\title{
A THIRD ORDER CORRECTION TO THE HELMHOLTZ EQUATION
}

\author{
J. JEGOROVS and J. MOHRING \\ Fraunhofer ITWM and Universität Kaiserslautern \\ Gottlieb-Daimler-Str. 49, D-67663, Kaiserslautern, Germany \\ E-mail: jegorovs@itwm.fhg.de; mohring@itwm.fhg.de
}

Received October 1, 2004; revised January 8, 2005

\begin{abstract}
In this work we derive a third order correction to the classical Helmholtz equation. Starting from non-linear Euler equations and using asymptotical analysis we get a decoupled system of linear, Helmholtz type equations, which are written in terms of the acoustical pressure functions. We present also a rather simple concept of the boundary conditions. Also numerical results and accompanying difficulties are discussed and presented.

Key words: non-linear acoustics, Euler equations, asymptotic analysis, scaling, Helmholtz type equation, pressure, displacement, Lagrangian coordinates, non-homogeneous Neumann boundary conditions, radiation boundary conditions
\end{abstract}

\section{Introduction}

If a bass loudspeaker is run at high power, owing to the low frequency, the displacements of the membrane and of the air in the reflex tube may exceed a centimeter. Hence, linear acoustic approximation is no longer applicable. Solving the complete Euler equations, however, requires an unreasonably high effort. Applying asymptotical analysis and assuming irrotational airflow one was able to derive a second order correction to the classical Helmholtz equation from the full Euler equations. This correction is again of Helmholtz type with non-homogeneous right hand side, which depends only on the first order solution and its derivatives, cf. [3]. To estimate the significance of higher order corrections at least the third order correction is needed. The model in [3] was based on Lagrangian coordinates, hence, the natural unknown function was the displacement function $\mathbf{h}(t, x)$, however, we are interested in the behaviour of the acoustical pressure function $p(t, x)$.

\section{Isentropic Euler Equations}

We start from the isentropic Euler equations written in Eulerian coordinate system: 


$$
\begin{aligned}
\frac{\partial \rho}{\partial \tilde{t}}+\operatorname{div}_{\tilde{\mathbf{x}}}(\rho \mathbf{u}) & =0 \quad \text { (conservation of mass), } \\
\rho\left[\frac{\partial \mathbf{u}}{\partial \tilde{t}}+\left\langle\mathbf{u}, \nabla_{\tilde{\mathbf{x}}}\right\rangle \mathbf{u}\right]+\nabla_{\tilde{\mathbf{x}}} p & =0 \quad \text { (conservation of momentum) } \\
\frac{p}{p_{0}}-\left(\frac{\rho}{\rho_{0}}\right)^{\gamma} & =0 \quad \text { (isentropic). }
\end{aligned}
$$

Density $\rho$, pressure $p$ and velocity $\mathbf{u}$ are functions of the time $\tilde{t}>0$ and the position $\tilde{\mathbf{x}} \in \Omega \subset \mathbb{R}^{3}$, where " $\sim$ " denotes Eulerian coordinates. $\gamma$ is the gas specific heat ratio. $\rho_{0}$ and $p_{0}$ are the values of density and pressure at rest, respectively. Boundary conditions will be discussed later.

In our case we consider a domain, which is changing in time. It happens due to oscillations of the membrane. Because of this we transform the equations (2.1)-(2.3) into Lagrangian coordinate system $(t, x)$. Such transformation allows us to introduce new unknown function $\mathbf{h}(t, x)$, which is called the displacement (see [3]). In Lagrangian coordinate system the equations (2.1)-(2.3) are transformed into the following equation:

$$
\boldsymbol{F}^{\mathrm{t}} \frac{\partial^{2} \mathbf{h}}{\partial t^{2}}=\frac{c_{0}^{2}}{1-\gamma} \nabla_{\mathbf{x}}[(\operatorname{det} \boldsymbol{F})]^{1-\gamma},
$$

where $\boldsymbol{F}=\left(\delta_{i j}+\frac{\partial h_{i}}{\partial x_{j}}\right)_{i, j=1}^{3}$ and $c_{0}$ is the speed of the sound at rest.

\subsection{Asymptotic Analysis and Expansions}

In order to do linearization we apply the asymptotic analysis. To do so, we have to scale the equation (2.4). We introduce new dimensionless variables $\boldsymbol{\xi}:=\kappa \mathbf{x}$, $\tau:=\omega t$ and the scaled displacement $\boldsymbol{\eta}:=\mathbf{h} / l$, where $\kappa$ is so-called wave number, $l$ is a characteristic amplitude of the membrane oscillations and $\omega$ is the angular frequency. We define some quantity $\varepsilon:=\kappa l$. This quantity for low $\omega$ frequencies has to be small compared to 1 . Using these new variables we scale the equation (2.4):

$$
\varepsilon F^{\mathrm{t}} \frac{\partial^{2} \boldsymbol{\eta}}{\partial \tau^{2}}=\frac{1}{1-\gamma} \nabla_{\xi}[(\operatorname{det} F)]^{1-\gamma}
$$

where $\boldsymbol{F}=\left(\delta_{i j}+\varepsilon \frac{\partial \eta_{i}}{\partial \xi_{j}}\right)_{i, j=1}^{3}$.

Assume, without any proof of the convergence, that the displacement function $\boldsymbol{\eta}(\tau, \boldsymbol{\xi})$ has an expansion of the form (perfect harmonic excitation of the membrane is assumed):

$$
\begin{aligned}
\boldsymbol{\eta}(\tau, \boldsymbol{\xi})= & \Re\left(\boldsymbol{\eta}_{1}(\boldsymbol{\xi}) e^{i \tau}\right)+\varepsilon \Re\left(\boldsymbol{\eta}_{2}(\boldsymbol{\xi}) e^{2 i \tau}+\boldsymbol{\theta}_{2}(\boldsymbol{\xi})\right) \\
& +\varepsilon^{2} \Re\left(\boldsymbol{\eta}_{3}(\boldsymbol{\xi}) e^{3 i \tau}+\boldsymbol{\theta}_{3}(\boldsymbol{\xi}) e^{i \tau}\right)+\mathcal{O}\left(\varepsilon^{3}\right)
\end{aligned}
$$

At the same time we also assume existence of the expansion for the pressure function $p(\tau, \boldsymbol{\xi})$, which will be frequently used in the following analysis: 


$$
\begin{aligned}
p(\tau, \boldsymbol{\xi})= & \rho_{0} c_{0}^{2} \Re\left[\frac{1}{\gamma}+\varepsilon p_{1}(\boldsymbol{\xi}) e^{i \tau}+\varepsilon^{2}\left(p_{2}(\boldsymbol{\xi}) e^{2 i \tau}+q_{2}(\boldsymbol{\xi})\right)\right. \\
& \left.+\varepsilon^{3}\left(p_{3}(\boldsymbol{\xi}) e^{3 i \tau}+q_{3}(\boldsymbol{\xi}) e^{i \tau}\right)\right]+\mathcal{O}\left(\varepsilon^{4}\right) .
\end{aligned}
$$

Substituting the expression (2.6) into the equation (2.5), collecting terms for equal powers of $\varepsilon$ and for equal time factors $e^{\mathrm{i} \tau}, e^{2 \mathrm{i} \tau}$ and $e^{3 i \tau}$ we end up with the following equations for the functions $\boldsymbol{\eta}_{1}, \boldsymbol{\eta}_{2}$ and $\boldsymbol{\eta}_{3}$ :

$$
\begin{aligned}
& \nabla_{\xi}\left\langle\nabla_{\xi}, \boldsymbol{\eta}_{1}\right\rangle+\boldsymbol{\eta}_{1}=0 \\
& \nabla_{\xi}\left\langle\nabla_{\xi}, \boldsymbol{\eta}_{2}\right\rangle+4 \boldsymbol{\eta}_{2}=\frac{\nabla_{\xi}}{4}\left[(\gamma-1)\left\langle\nabla_{\xi}, \boldsymbol{\eta}_{1}\right\rangle^{2}-\left\langle\boldsymbol{\eta}_{1}, \boldsymbol{\eta}_{1}\right\rangle+\frac{\partial \eta_{1 j}}{\partial \xi_{k}} \frac{\partial \eta_{1 k}}{\partial \xi_{j}}\right] \\
& \nabla_{\xi}\left\langle\nabla_{\xi}, \boldsymbol{\eta}_{3}\right\rangle+9 \boldsymbol{\eta}_{3}=\frac{\nabla_{\xi}}{2}\left[-\frac{(\gamma-1)^{2}}{12}\left\langle\nabla_{\xi}, \boldsymbol{\eta}_{1}\right\rangle^{3}-\left\langle\boldsymbol{\eta}_{1}, \boldsymbol{\eta}_{2}\right\rangle\right. \\
& -\frac{\gamma-1}{4}\left\langle\nabla_{\xi}, \boldsymbol{\eta}_{1}\right\rangle \frac{\partial \eta_{1 j}}{\partial \xi_{k}} \frac{\partial \eta_{1 k}}{\partial \xi_{j}}-\frac{1}{6} \frac{\partial \eta_{1 j}}{\partial \xi_{k}} \frac{\partial \eta_{1 k}}{\partial \xi_{m}} \frac{\partial \eta_{1 m}}{\partial \xi_{j}}+\frac{\partial \eta_{1 j}}{\partial \xi_{k}} \frac{\partial \eta_{2 k}}{\partial \xi_{j}} \\
& \left.+(\gamma-1)\left\langle\nabla_{\xi}, \boldsymbol{\eta}_{1}\right\rangle\left\langle\nabla_{\xi}, \boldsymbol{\eta}_{2}\right\rangle\right]-\frac{3}{2}\left(\frac{\partial \eta_{1 j}}{\partial \xi_{1}} \eta_{2 j}, \frac{\partial \eta_{1 j}}{\partial \xi_{2}} \eta_{2 j}, \frac{\partial \eta_{1 j}}{\partial \xi_{3}} \eta_{2 j}\right)^{\mathrm{T}} .
\end{aligned}
$$

Here the Einstein's summation convention is assumed.

\subsection{Non-existence of the potential of $\eta_{3}$}

Assume for the moment, that the functions $\boldsymbol{\eta}_{1}, \boldsymbol{\eta}_{2}$ have potentials, i.e. $\exists \phi_{1}$ and $\phi_{2}$ such that $\boldsymbol{\eta}_{1}=\nabla_{\xi} \phi_{1}$ and $\boldsymbol{\eta}_{2}=\nabla_{\xi} \phi_{2}$. Inserting these expressions into the equations (2.8) and (2.9) and integrating both left and right hand sides of the resulting expressions with respect to $\xi_{i}, i=1,2,3$ (assume the integration constants equal to zero) we end up with the equations for the functions $\phi_{1}$ and $\phi_{2}$ :

$$
\begin{aligned}
\Delta_{\xi} \phi_{1}+\phi_{1} & =0 \\
\Delta_{\xi} \phi_{2}+4 \phi_{2} & =\frac{1}{4}\left[(\gamma-1) \phi_{1}^{2}-\left(\nabla_{\xi} \phi_{1}\right)^{2}+\left(\frac{\partial^{2} \phi_{1}}{\partial \xi_{j} \partial \xi_{k}}\right)^{2}\right] .
\end{aligned}
$$

These are exactly the equations described in [3], which are the basis for the equations written in terms of the functions $p_{1}$ and $p_{2}$ what we are interested in.

Assume the existence of a potential of the function $\boldsymbol{\eta}_{3}$, i.e. $\boldsymbol{\eta}_{3}=\nabla_{\xi} \phi_{3}$, and try to integrate the right hand side of the equation (2.10) with respect to $\xi_{i}, i=1,2,3$. The last term of the right hand side of the equation (2.10) after the integration, in general, gives three different results depending on the direction of the integration:

$$
\int \frac{\partial^{2} \phi_{1}}{\partial \xi_{1} \partial \xi_{j}} \frac{\partial \phi_{2}}{\partial \xi_{j}} d \xi_{1} \neq \int \frac{\partial^{2} \phi_{1}}{\partial \xi_{2} \partial \xi_{j}} \frac{\partial \phi_{2}}{\partial \xi_{j}} d \xi_{2} \neq \int \frac{\partial^{2} \phi_{1}}{\partial \xi_{3} \partial \xi_{j}} \frac{\partial \phi_{2}}{\partial \xi_{j}} d \xi_{3} .
$$


This means that we have got a contradiction (according to Poincaré's lemma, cf. [1]) and $\boldsymbol{\eta}_{3}$ could not have a potential. Hence, we cannot derive the equation for the function $\phi_{3}$ and, hence, we cannot derive the equation for the function $p_{3}$ using the same technique as in [3].

Remark 1. Another proof of non-existence of the potential of the function $\eta_{3}$ could be done if we apply the rot operator to the equation (2.10) and investigate the properties of this new equality.

\subsection{Third order correction}

In order to derive the equation for the function $p_{3}$ avoiding the difficulties discussed above, we consider isentropic state equation written in Lagrangian coordinates, cf. [3]:

$$
p=\frac{\rho_{0} c_{0}^{2}}{\gamma}(\operatorname{det} F)^{-\gamma} .
$$

Rewriting the right hand side of the equation (2.13) in terms of $\boldsymbol{\eta}_{1}, \boldsymbol{\eta}_{2}$ and $\boldsymbol{\eta}_{3}$, collecting the terms of equal powers of $\varepsilon$ and time factors, and comparing with the relation (2.7), we end up with the relations for the functions $p_{1}, p_{2}$ and $p_{3}$ :

$$
\begin{aligned}
p_{1} & =-\left\langle\nabla_{\xi}, \boldsymbol{\eta}_{1}\right\rangle \\
p_{2} & =-\left\langle\nabla_{\xi}, \boldsymbol{\eta}_{2}\right\rangle+\frac{\gamma}{4}\left\langle\nabla_{\xi}, \boldsymbol{\eta}_{1}\right\rangle^{2}+\frac{1}{4} \frac{\partial \eta_{1 j}}{\partial \xi_{k}} \frac{\partial \eta_{1 k}}{\partial \xi_{j}} \\
p_{3} & =-\left\langle\nabla_{\xi}, \boldsymbol{\eta}_{3}\right\rangle+\frac{\gamma}{2}\left\langle\nabla_{\xi}, \boldsymbol{\eta}_{1}\right\rangle\left\langle\nabla_{\xi}, \boldsymbol{\eta}_{2}\right\rangle+\frac{1}{2} \frac{\partial \eta_{1 j}}{\partial \xi_{k}} \frac{\partial \eta_{2 k}}{\partial \xi_{j}}-\frac{\gamma^{2}}{24}\left\langle\nabla_{\xi}, \boldsymbol{\eta}_{1}\right\rangle^{3} \\
& -\frac{\gamma}{8} \frac{\partial \eta_{1 j}}{\partial \xi_{k}} \frac{\partial \eta_{1 k}}{\partial \xi_{j}}\left\langle\nabla_{\xi}, \boldsymbol{\eta}_{1}\right\rangle-\frac{1}{12} \frac{\partial \eta_{1 j}}{\partial \xi_{k}} \frac{\partial \eta_{1 k}}{\partial \xi_{m}} \frac{\partial \eta_{1 m}}{\partial \xi_{j}}
\end{aligned}
$$

Applying the gradient operator to the relations (2.14), (2.15) and (2.16), taking into account the equations (2.8), (2.9) and (2.10), we get the expressions for the functions $\boldsymbol{\eta}_{1}, \boldsymbol{\eta}_{2}$ and $\boldsymbol{\eta}_{3}$ written in terms of $p_{1}, p_{2}$ and $p_{3}$. Inserting these relations into the equations (2.8), (2.9) and (2.10) and integrating the results with respect to $\xi_{i}, i=$ $1,2,3$ (again, we assume that the integration constants are zeros) we end up with the equations for the functions $p_{1}, p_{2}$ and $p_{3}$ :

$$
\begin{aligned}
\Delta_{\xi} p_{1}+p_{1} & =0 \\
\Delta_{\xi} p_{2}+4 p_{2} & =\left(\gamma-\frac{1}{2}\right) p_{1}^{2}+\frac{3}{2}\left(\frac{\partial^{2} p_{1}}{\partial \xi_{j} \partial \xi_{k}}\right)^{2} \\
\Delta_{\xi} p_{3}+9 p_{3} & =-\frac{3\left(\gamma-\frac{1}{2}\right)\left(\gamma-\frac{1}{3}\right)}{4} p_{1}^{3}+\frac{9 \gamma-5}{2} p_{1} p_{2}-\frac{1}{8} p_{1}\left(\frac{\partial^{2} p_{1}}{\partial \xi_{j} \partial \xi_{k}}\right)^{2} \\
& +\frac{7}{4} \frac{\partial^{2} p_{1}}{\partial \xi_{j} \partial \xi_{k}} \frac{\partial^{2} p_{2}}{\partial \xi_{j} \partial \xi_{k}}-\frac{5}{8} \frac{\partial p_{1}}{\partial \xi_{j}} \frac{\partial^{2} p_{1}}{\partial \xi_{k} \partial \xi_{m}} \frac{\partial^{3} p_{1}}{\partial \xi_{j} \partial \xi_{k} \partial \xi_{m}} \\
& -\frac{13}{8} \frac{\partial^{2} p_{1}}{\partial \xi_{j} \partial \xi_{k}} \frac{\partial^{2} p_{1}}{\partial \xi_{k} \partial \xi_{m}} \frac{\partial^{2} p_{1}}{\partial \xi_{m} \partial \xi_{j}}-\frac{5}{8} \frac{\partial p_{1}}{\partial \xi_{j}} \frac{\partial p_{1}}{\partial \xi_{k}} \frac{\partial^{2} p_{1}}{\partial \xi_{j} \partial \xi_{k}}
\end{aligned}
$$


In dimensional form these equations look like:

$$
\begin{aligned}
\Delta_{\mathbf{x}} p_{1}+\kappa^{2} p_{1} & =0 \\
\Delta_{\mathbf{x}} p_{2}+4 \kappa^{2} p_{2} & =\left(\gamma-\frac{1}{2}\right) \kappa^{2} p_{1}^{2}+\frac{3}{2 \kappa^{2}}\left(\frac{\partial^{2} p_{1}}{\partial x_{j} \partial x_{k}}\right)^{2} \\
\Delta_{\mathbf{x}} p_{3}+9 \kappa^{2} p_{3} & =\kappa^{2} p_{1}\left[-\frac{3\left(\gamma-\frac{1}{2}\right)\left(\gamma-\frac{1}{3}\right)}{4} p_{1}^{2}+\frac{9 \gamma-5}{2} p_{2}-\frac{1}{8 \kappa^{4}}\left(\frac{\partial^{2} p_{1}}{\partial x_{j} \partial x_{k}}\right)^{2}\right] \\
& +\frac{7}{4 \kappa^{2}} \frac{\partial^{2} p_{1}}{\partial x_{j} \partial x_{k}} \frac{\partial^{2} p_{2}}{\partial x_{j} \partial x_{k}}-\frac{13}{8 \kappa^{4}} \frac{\partial^{2} p_{1}}{\partial x_{j} \partial x_{k}} \frac{\partial^{2} p_{1}}{\partial x_{k} \partial x_{m}} \frac{\partial^{2} p_{1}}{\partial x_{m} \partial x_{j}} \\
& -\frac{5}{8 \kappa^{2}} \frac{\partial p_{1}}{\partial x_{j}} \frac{\partial p_{1}}{\partial x_{k}} \frac{\partial^{2} p_{1}}{\partial x_{j} \partial x_{k}}-\frac{5}{8 \kappa^{4}} \frac{\partial p_{1}}{\partial x_{j}} \frac{\partial^{2} p_{1}}{\partial x_{k} \partial x_{m}} \frac{\partial^{3} p_{1}}{\partial x_{j} \partial x_{k} \partial x_{m}} .
\end{aligned}
$$

We have to note that equations (2.20) and (2.21) were derived in [3] using the assumption that $\boldsymbol{\eta}_{1}$ and $\boldsymbol{\eta}_{2}$ have potentials. In the present derivation this assumption is relaxed and, as consequence, we were able to derive the third order correction to the homogeneous Helmholtz equation in terms of the pressure. The equation (2.22) is of Helmholtz type with non-homogeneous free term, which depends on the first and second order solutions.

Completely in the same manner we can derive the equations for the functions $q_{2}$ and $q_{3}$ (non-dimensional form):

$$
\begin{aligned}
q_{2} & =\frac{1}{4}\left|p_{1}\right|^{2}+\frac{1}{4}\left|\frac{\partial p_{1}}{\partial \xi_{j}}\right|^{2}, \\
\Delta_{\xi} q_{3}+q_{3} & =\frac{-2 \gamma^{2}+9 \gamma-5}{8} p_{1}\left|p_{1}\right|^{2}+\frac{\gamma-5}{2} p_{2} \bar{p}_{1}+\frac{3}{4} \bar{p}_{1}\left(\frac{\partial^{2} p_{1}}{\partial \xi_{j} \partial \xi_{k}}\right)^{2} \\
& +\frac{\gamma-1}{4} p_{1}\left|\frac{\partial p_{1}}{\partial \xi_{j}}\right|^{2}+\frac{3}{4} \frac{\partial^{2} p_{2}}{\partial \xi_{j} \partial \xi_{k}} \frac{\partial^{2} \bar{p}_{1}}{\partial \xi_{j} \partial \xi_{k}}+\frac{1}{2} \frac{\partial p_{1}}{\partial \xi_{j}} \frac{\partial \bar{p}_{1}}{\partial \xi_{k}} \frac{\partial^{2} p_{1}}{\partial \xi_{j} \partial \xi_{k}} \\
& -\frac{1}{8} \frac{\partial p_{1}}{\partial \xi_{j}} \frac{\partial p_{1}}{\partial \xi_{k}} \frac{\partial^{2} \bar{p}_{1}}{\partial \xi_{j} \partial \xi_{k}}+\frac{1}{8} p_{1}\left|\frac{\partial^{2} p_{1}}{\partial \xi_{j} \partial \xi_{k}}\right|^{2}+2 \frac{\partial^{2} p_{1}}{\partial \xi_{j} \partial \xi_{k}} \frac{\partial^{2} s_{2}}{\partial \xi_{j} \partial \xi_{k}} \\
& -\frac{5}{8} \frac{\partial^{2} p_{1}}{\partial \xi_{j} \partial \xi_{k}} \frac{\partial^{2} p_{1}}{\partial \xi_{k} \partial \xi_{m}} \frac{\partial^{2} \bar{p}_{1}}{\partial \xi_{m} \partial \xi_{j}}+\frac{1}{4} \frac{\partial \bar{p}_{1}}{\partial \xi_{j}} \frac{\partial^{2} p_{1}}{\partial \xi_{k} \partial \xi_{m}} \frac{\partial^{3} p_{1}}{\partial \xi_{j} \partial \xi_{k} \partial \xi_{m}} \\
& -\frac{1}{8} \frac{\partial p_{1}}{\partial \xi_{j}} \frac{\partial^{2} \bar{p}_{1}}{\partial \xi_{k} \partial \xi_{m}} \frac{\partial^{3} p_{1}}{\partial \xi_{j} \partial \xi_{k} \partial \xi_{m}}+\frac{1}{4} \frac{\partial p_{1}}{\partial \xi_{j}} \frac{\partial^{2} p_{1}}{\partial \xi_{k} \partial \xi_{m}} \frac{\partial^{3} \bar{p}_{1}}{\partial \xi_{j} \partial \xi_{k} \partial \xi_{m}} .
\end{aligned}
$$

Here $s_{2}$ function is a potential of the function $\boldsymbol{\theta}_{2}$. We made this assumption in order to simplify our derivation, and it has to be proved yet. $s_{2}$ is an auxiliary quantity and it is the solution of the Poisson equation, where the right hand side depends on the function $p_{1}$ and its derivatives:

$$
\Delta_{\xi} s_{2}=\frac{\gamma-1}{4}\left|p_{1}\right|^{2}-\frac{1}{4}\left|\frac{\partial p_{1}}{\partial \xi_{j}}\right|^{2}+\frac{1}{4}\left|\frac{\partial^{2} p_{1}}{\partial \xi_{j} \xi_{k}}\right|^{2} .
$$




\section{Boundary Conditions}

In the previous section we derived the equations for unknown functions $p_{1}, p_{2}$ and $p_{3}$. In order to complete the model we have to prescribe boundary conditions, which are completely consistent up to the orders of our approximation and the geometry. So far we mentioned, that the domain $\Omega \subset \mathbb{R}^{3}$ in Eulerian description changes its shape. To keep $\Omega$ fixed we introduced Lagrangian coordinates. The computational domain consists of two main parts: the first part is a bass loudspeaker with cylindrical symmetry itself $\left(\Omega_{l}\right)$ and the second part is an artificial region $\left(\Omega_{a}\right)$, cf. Fig. 1 .

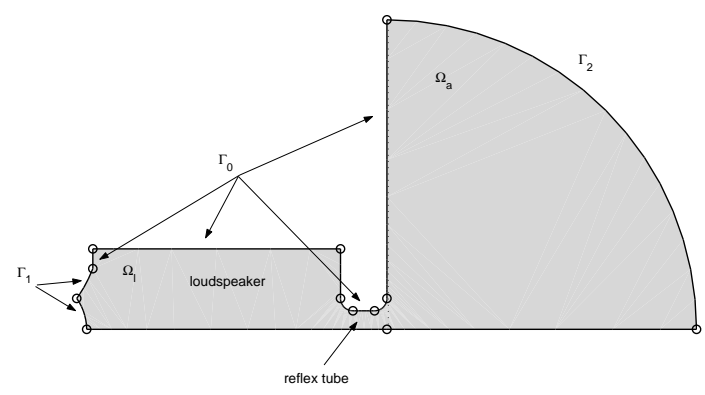

Figure 1. Computational domain.

Also, we distinguish three kinds of boundaries of the domain $\Omega$ : boundaries which are fixed $\left(\Gamma_{0}\right)$, harmonically oscillating boundaries with amplitude $\boldsymbol{\eta}$ at angular frequency 1 (respectively $l \boldsymbol{\eta}$ and $\omega$ in dimensional variables) $\left(\Gamma_{1}\right)$ and segments of spheres of radius $\varrho_{s}$ where sound is radiated through $\left(\Gamma_{2}\right)$. Because our bass loudspeaker has axial symmetry it is enough to consider $2 \mathrm{D}$ boundaries. Also we restrict ourselves to special case: circular boundaries.

\subsection{Circular boundaries}

In this subsection we consider the case of the circular boundaries. The reason is simple: almost all parts of the boundaries of $\Omega$ have circular shape, i.e. they can be represented as parts of a circle. A straight line could be represented as a part of the circle with infinite radius. From now on we will not make a difference between circles and straight lines.

Let us consider some part of the circle of some fixed radius $\varrho$ and denote this part by $\Gamma$, cf. Fig. $2 a$. Let us denote the position of the center of this circle by $\boldsymbol{\xi}_{0}$. Consider at some point on $\Gamma$ a small control volume $\Omega_{0}$. Now, we assume that $\Gamma$ is periodically oscillating with frequency 1 along vector $\nu$, cf. Fig. $2 b$. During the oscillations the center of the circle is also oscillating ( $\varrho$ is fixed). So, we may express the position of the center by $\boldsymbol{\xi}_{0}+\boldsymbol{\eta}_{0}(\tau)$, where $\boldsymbol{\eta}_{0}(\tau)$ is given and assumed to be $\boldsymbol{\nu} \Re\left(e^{i \tau}\right)$. The control volume $\Omega_{0}$, due to oscillations of $\Gamma$ and due to very small 


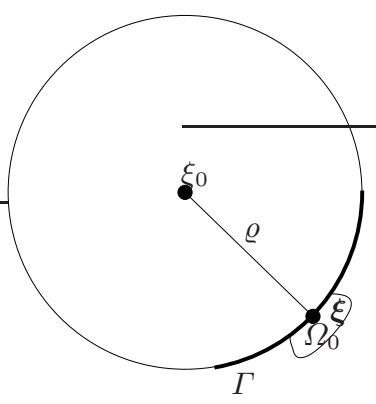

a)

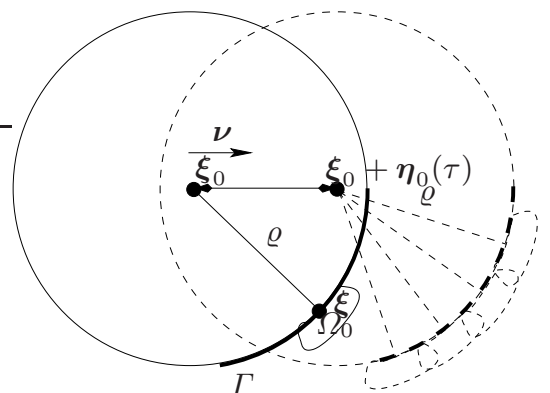

b)

Figure 2. The behaviour of the circular boundary.

viscosity of the air, is moving along $\Gamma$ (control volume on $\Gamma$ sticks to it). This is an assumption. So, we do not know, the position of this control volume, but we know, that the distance between the center of the circle and the control volume is always constant, see Fig. $2 b$. Assume, that the control volume moves from point $\xi$ to the point $\boldsymbol{\xi}+\boldsymbol{\eta}(\tau, \boldsymbol{\xi})$. In that case the distance between the control volume and the center of the circle is constant, i.e.:

$$
\left|\boldsymbol{\xi}+\varepsilon \boldsymbol{\eta}(\tau, \boldsymbol{\xi})-\boldsymbol{\xi}_{0}-\varepsilon \boldsymbol{\eta}_{0}(\tau)\right|^{2}=\varrho^{2} .
$$

Simplifying this relation we get:

$$
\left\langle\boldsymbol{n}, \boldsymbol{\eta}(\tau, \boldsymbol{\xi})-\boldsymbol{\eta}_{0}(\tau)\right\rangle=\frac{\varepsilon\left|\boldsymbol{\eta}(\tau, \boldsymbol{\xi})-\boldsymbol{\eta}_{0}(\tau)\right|^{2}}{2 \varrho} .
$$

Inserting the expansion (2.6) into relation (3.2), collecting corresponding terms we end up with the boundary conditions for the functions $\boldsymbol{\eta}_{1}, \boldsymbol{\eta}_{2}$ and $\boldsymbol{\eta}_{3}$ :

$$
\begin{aligned}
& \left\langle\boldsymbol{n}, \boldsymbol{\eta}_{1}\right\rangle=\langle\boldsymbol{n}, \boldsymbol{\nu}\rangle, \\
& \left\langle\boldsymbol{n}, \boldsymbol{\eta}_{2}\right\rangle=\frac{1}{4 \varrho}\left(\boldsymbol{\eta}_{1}-\boldsymbol{\nu}\right)^{2}, \\
& \left\langle\boldsymbol{n}, \boldsymbol{\eta}_{3}\right\rangle=\frac{1}{2 \varrho}\left\langle\boldsymbol{\eta}_{1}-\boldsymbol{\nu}, \boldsymbol{\eta}_{2}\right\rangle .
\end{aligned}
$$

Remembering the expressions of $\boldsymbol{\eta}_{1}, \boldsymbol{\eta}_{2}$ and $\boldsymbol{\eta}_{3}$ written in terms of $p_{1}, p_{2}$ and $p_{3}$ we end up with the boundary conditions for functions $p_{1}, p_{2}$ and $p_{3}$ listed in Tab. 1 . The radiation condition means that the sound waves behave as if there were point sources in the aperture of frequency 1 for $p_{1}$, frequency 2 for $p_{2}$ and frequency 3 for $p_{3}$. It is commonly used in linear acoustics, but has to be justified more thoroughly for $p_{2}$ and $p_{3}$ yet. 
Table 1. Boundary conditions.

\begin{tabular}{cccc}
\hline & $\Gamma_{0}$ & $\Gamma_{1}$ & $\Gamma_{2}$ \\
\hline$p_{1}$ & $\frac{\partial p_{1}}{\partial \boldsymbol{n}}=0$ & $\frac{\partial p_{1}}{\partial \boldsymbol{n}}=\langle\boldsymbol{n}, \boldsymbol{\nu}\rangle$ & $\frac{\partial p_{1}}{\partial \boldsymbol{n}}+\left(i+\varrho_{s}^{-1}\right) p_{1}=0$ \\
$p_{2}$ & $\frac{\partial p_{2}}{\partial \boldsymbol{n}}=A(\mathbf{0})$ & $\frac{\partial p_{2}}{\partial \boldsymbol{n}}=A(\boldsymbol{\nu})$ & $\frac{\partial p_{2}}{\partial \boldsymbol{n}}+\left(2 i+\varrho_{s}^{-1}\right) p_{2}=0$ \\
$p_{3}$ & $\frac{\partial p_{3}}{\partial \boldsymbol{n}}=B(\mathbf{0})$ & $\frac{\partial p_{3}}{\partial \boldsymbol{n}}=B(\boldsymbol{\nu})$ & $\frac{\partial p_{3}}{\partial \boldsymbol{n}}+\left(3 i+\varrho_{s}^{-1}\right) p_{3}=0$ \\
\hline
\end{tabular}

In Tab. 1 we use notation:

$$
\begin{aligned}
A(\boldsymbol{\nu}) & :=\frac{1}{\varrho}\left(\nabla_{\xi} p_{1}-\boldsymbol{\nu}\right)^{2}+\frac{1}{4}\left\langle\boldsymbol{n}, \nabla_{\xi}\left(p_{1}^{2}\right)+\nabla_{\xi}\left(\left(\nabla_{\xi} p_{1}\right)^{2}\right)\right\rangle \\
B(\boldsymbol{\nu}) & :=\left\langle\boldsymbol{n}, \frac{1}{2} \nabla_{\xi}\left(p_{1} p_{2}\right)-\frac{\gamma+1}{24} \nabla_{\xi}\left(p_{1}^{3}\right)+\frac{1}{16}\left[8 \frac{\partial^{2} p_{1}}{\partial \xi_{i} \partial \xi_{j}} \frac{\partial p_{2}}{\partial \xi_{j}}\right.\right. \\
& +2 \frac{\partial^{2} p_{2}}{\partial \xi_{i} \partial \xi_{j}} \frac{\partial p_{1}}{\partial \xi_{j}}-\frac{\partial p_{1}}{\partial \xi_{i}}\left(\frac{\partial p_{1}}{\partial \xi_{j}}\right)^{2}-5 \frac{\partial p_{1}}{\partial \xi_{j}} \frac{\partial^{2} p_{1}}{\partial \xi_{j} \partial \xi_{i}} p_{1} \\
& \left.\left.-5 \frac{\partial^{2} p_{1}}{\partial \xi_{j} \partial \xi_{i}} \frac{\partial p_{1}}{\partial \xi_{k}} \frac{\partial^{2} p_{1}}{\partial \xi_{j} \partial \xi_{k}}-\frac{\partial p_{1}}{\partial \xi_{j}} \frac{\partial p_{1}}{\partial \xi_{k}} \frac{\partial^{3} p_{1}}{\partial \xi_{j} \partial \xi_{k} \partial \xi_{i}}\right]\right\rangle \\
& +\frac{9}{8 \varrho}\left\langle\nabla_{\xi} p_{1}-\boldsymbol{\nu}, \nabla_{\xi} p_{2}-\frac{1}{4} \nabla_{\xi}\left(p_{1}^{2}\right)-\frac{1}{4} \nabla_{\xi}\left(\left(\nabla_{\xi} p_{1}\right)^{2}\right)\right\rangle,
\end{aligned}
$$

in non-dimensional form, and

$$
\begin{aligned}
A(\boldsymbol{\nu}) & :=\frac{1}{\kappa^{3} r}\left(\nabla_{\mathbf{x}} p_{1}-\kappa \boldsymbol{\nu}\right)^{2}+\frac{1}{4 \kappa}\left\langle\boldsymbol{n}, \nabla_{\mathbf{x}}\left(p_{1}^{2}\right)+\frac{1}{\kappa^{2}} \nabla_{\mathbf{x}}\left(\left(\nabla_{\mathbf{x}} p_{1}\right)^{2}\right)\right\rangle \\
B(\boldsymbol{\nu}) & :=\left\langle\boldsymbol{n}, \frac{1}{2 \kappa} \nabla_{\mathbf{x}}\left(p_{1} p_{2}\right)-\frac{\gamma+1}{24 \kappa} \nabla_{\mathbf{x}}\left(p_{1}^{3}\right)+\frac{1}{16 \kappa^{3}}\left[8 \frac{\partial^{2} p_{1}}{\partial x_{i} \partial x_{j}} \frac{\partial p_{2}}{\partial x_{j}}\right.\right. \\
& +2 \frac{\partial^{2} p_{2}}{\partial x_{i} \partial x_{j}} \frac{\partial p_{1}}{\partial x_{j}}-\frac{\partial p_{1}}{\partial x_{i}}\left(\frac{\partial p_{1}}{\partial x_{j}}\right)^{2}-5 \frac{\partial p_{1}}{\partial x_{j}} \frac{\partial^{2} p_{1}}{\partial x_{j} \partial x_{i}} p_{1} \\
& \left.\left.-\frac{5}{\kappa^{2}} \frac{\partial^{2} p_{1}}{\partial x_{j} \partial x_{i}} \frac{\partial p_{1}}{\partial x_{k}} \frac{\partial^{2} p_{1}}{\partial x_{j} \partial x_{k}}-\frac{1}{\kappa^{2}} \frac{\partial p_{1}}{\partial x_{j}} \frac{\partial p_{1}}{\partial x_{k}} \frac{\partial^{3} p_{1}}{\partial x_{j} \partial x_{k} \partial x_{i}}\right]\right\rangle \\
& +\frac{9}{8 \kappa^{3} r}\left\langle\nabla_{\mathbf{x}} p_{1}-\kappa \boldsymbol{\nu}, \nabla_{\mathbf{x}} p_{2}-\frac{1}{4} \nabla_{\mathbf{x}}\left(p_{1}^{2}\right)-\frac{1}{4 \kappa^{2}} \nabla_{\mathbf{x}}\left(\left(\nabla_{\mathbf{x}} p_{1}\right)^{2}\right)\right\rangle
\end{aligned}
$$

in dimensional variables, where $r$ is non-scaled radius.

We have specified the Neumann boundary conditions for functions $p_{1}, p_{2}$ and $p_{3}$ on the moving and non-moving boundaries. Not always these conditions are homogeneous. Especially, let us note, that the conditions on the curved non-moving 
boundaries are non-homogeneous in general. In next section we will show numerically that naive guess, that on the non-moving boundaries homogeneous Neumann boundary conditions (isolation conditions) are right choice, is wrong.

To complete this section we also present boundary conditions for the functions $s_{2}$ and $q_{3}$ written in non-dimensional form, cf. Tab. 2.

Table 2. Boundary conditions.

\begin{tabular}{|c|c|c|c|}
\hline & $\Gamma_{0}$ & $\Gamma_{1}$ & $\Gamma_{2}$ \\
\hline$s_{2}$ & $\frac{\partial s_{2}}{\partial \boldsymbol{n}}=C(\mathbf{0})$ & $\frac{\partial s_{2}}{\partial \boldsymbol{n}}=C(\boldsymbol{\nu})$ & $\frac{\partial s_{2}}{\partial \boldsymbol{n}}+\varrho_{s}^{-1} s_{2}=0$ \\
\hline$q_{3}$ & $\frac{\partial q_{3}}{\partial \boldsymbol{n}}=D(\mathbf{0})$ & $\frac{\partial q_{3}}{\partial \boldsymbol{n}}=D(\boldsymbol{\nu})$ & $\frac{\partial q_{3}}{\partial \boldsymbol{n}}+\left(i+\varrho_{s}^{-1}\right) q_{3}=0$ \\
\hline
\end{tabular}

Here we use notation

$$
\begin{aligned}
C(\boldsymbol{\nu}) & :=\frac{1}{4 \varrho}\left|\nabla_{\xi} p_{1}-\boldsymbol{\nu}\right|^{2}, \\
D(\boldsymbol{\nu}) & :=\left\langle\boldsymbol{n}, \frac{1-\gamma}{8} \nabla_{\xi}\left(p_{1}\left|p_{1}\right|^{2}\right)+\frac{1}{2} \frac{\partial^{2} \bar{p}_{1}}{\partial \xi_{i} \partial \xi_{j}} \frac{\partial p_{2}}{\partial \xi_{j}}+\frac{1}{8} \frac{\partial \bar{p}_{1}}{\partial \xi_{j}} \frac{\partial^{2} p_{2}}{\partial \xi_{i} \partial \xi_{j}}\right. \\
& +\frac{3}{16} \frac{\partial p_{1}}{\partial \xi_{i}}\left|\nabla_{\xi} p_{1}\right|^{2}+\frac{3}{16} p_{1} \frac{\partial \bar{p}_{1}}{\partial \xi_{j}} \frac{\partial^{2} p_{1}}{\partial \xi_{i} \partial \xi_{j}}-\frac{1}{16} \frac{\partial \bar{p}_{1}}{\partial \xi_{k}} \frac{\partial^{2} p_{1}}{\partial \xi_{j} \partial \xi_{k}} \frac{\partial^{2} p_{1}}{\partial \xi_{i} \partial \xi_{j}} \\
& \left.-\frac{1}{4} \frac{\partial p_{1}}{\partial \xi_{k}} \frac{\partial^{2} \bar{p}_{1}}{\partial \xi_{j} \partial \xi_{k}} \frac{\partial^{2} p_{1}}{\partial \xi_{i} \partial \xi_{j}}-\frac{1}{16} \frac{\partial p_{1}}{\partial \xi_{j}} \frac{\partial \bar{p}_{1}}{\partial \xi_{k}} \frac{\partial^{3} p_{1}}{\partial \xi_{i} \partial \xi_{j} \partial \xi_{k}}+\frac{\partial p_{1}}{\partial \xi_{j}} \frac{\partial^{2} s_{2}}{\partial \xi_{i} \partial \xi_{j}}\right\rangle \\
& +\frac{1}{2 \varrho}\left(2\left\langle\nabla_{\xi} p_{1}-\boldsymbol{\nu}, \nabla_{\xi} s_{2}\right\rangle+\frac{1}{4}\left\langle\nabla_{\xi} p_{1}-\boldsymbol{\nu}, \nabla_{\xi} p_{2}\right\rangle-\frac{1}{8} p_{1}\left|\nabla_{\xi} p_{1}\right|^{2}\right. \\
& \left.-\frac{1}{8} \frac{\partial p_{1}}{\partial \xi_{j}} \frac{\partial \bar{p}_{1}}{\partial \xi_{k}} \frac{\partial^{2} p_{1}}{\partial \xi_{j} \partial \xi_{k}}\right) .
\end{aligned}
$$

\section{Numerical Results}

For the simulation we used FEMLAB package, which uses the standard numerical methods based on the finite elements. In order to make our results comparable with physical experiments, we simulate the behaviour of the so-called Sound Pressure Level (SPL, [dB]). The formula is the following, cf. [5]:

$$
\mathrm{SPL}=10 \log _{10}\left(\frac{\frac{1}{T} \int_{0}^{T} p_{\mathrm{tot}}^{2}(t, \boldsymbol{x}) d t}{p_{0}^{2}}\right),
$$


where $T$ is certain time period, $p_{0}$ the pressure field at rest and $p_{\text {tot }}$ is given by

$p_{\text {tot }}(t, \boldsymbol{x})=\rho_{0} c_{0}^{2} \Re\left(\mu p_{1}(\boldsymbol{x}) e^{i \omega t}+\mu^{2} p_{2}(\boldsymbol{x}) e^{2 i \omega t}+\mu^{3}\left(p_{3}(\boldsymbol{x}) e^{3 i \omega t}+q_{3}(\boldsymbol{x}) e^{i \omega t}\right)\right)$,

where $\mu$ is undetermined quantity. Let us find $p_{\text {tot }}^{2}$ and insert the resulting expression into (4.1):

$$
\mathrm{SPL}=10 \log _{10}\left(\frac{\rho_{0}^{2} c_{0}^{4}}{2 p_{0}^{2}}\left(\mu^{2}\left|p_{1}+\mu^{2} q_{3}\right|^{2}+\mu^{4}\left|p_{2}\right|^{2}+\mu^{6}\left|p_{3}\right|^{2}\right)\right) .
$$

In the last expression the only unknown quantity is $\mu$ (SPL is some prescribed value). In order to find $\mu$ we consider the behaviour of the pressure components $p_{1}, p_{2}, p_{3}$ and $q_{3}$ on the boundary $\Gamma_{3}$, cf. Fig. 1 . Actually, we find the average values of $p_{1}, p_{2}$, $p_{3}$ and $q_{3}$ on $\Gamma_{3}$, i.e.:

$$
\bar{p}_{i}^{2}=\frac{\frac{1}{\left|\Gamma_{3}\right|} \int_{\left|\Gamma_{3}\right|} r\left|p_{i}\right|^{2} d r}{\int_{\left|\Gamma_{3}\right|} r d r}, \quad \bar{q}_{3}^{2}=\frac{\frac{1}{\left|\Gamma_{3}\right|} \int_{\left|\Gamma_{3}\right|} r\left|q_{3}\right|^{2} d r}{\int_{\left|\Gamma_{3}\right|} r d r}, \quad i=1,2,3,
$$

and insert them into (4.2). Hence, $\mu$ can be determined. The results of the simulation for SPL $=100[\mathrm{~dB}]$ on the $\Gamma_{3}$ and $\omega=65[\mathrm{~Hz}]$ are depicted in Fig. 3.

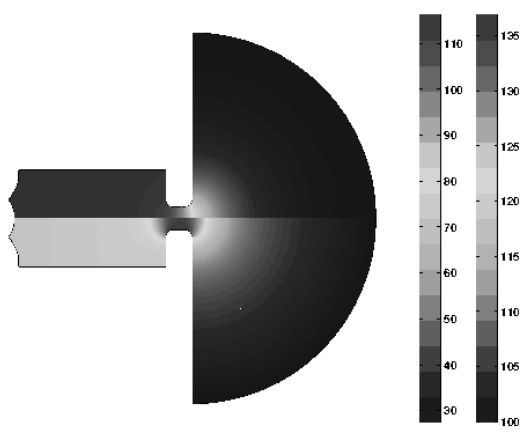

a)

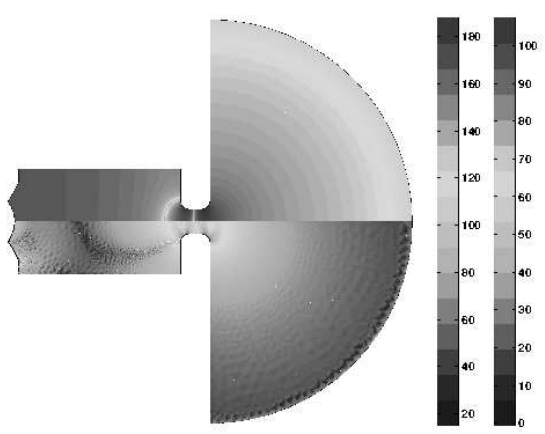

b)

Figure 3. a) The behaviour of $10 \log _{10}\left(\frac{\rho_{0}^{2} c_{0}^{4}}{2 p_{0}^{2}} \mu^{2}\left|p_{1}\right|^{2}\right)$ on the upper part (right color bar), $10 \log _{10}\left(\frac{\rho_{0}^{2} c_{0}^{4}}{2 p_{0}^{2}} \mu^{4}\left|p_{2}\right|^{2}\right)$ on the lower part (left color bar), b) the behaviour of $10 \log _{10}\left(\frac{\rho_{0}^{2} c_{0}^{4}}{2 p_{0}^{2}} \mu^{6}\left|p_{3}\right|^{2}\right)$ function (upper part) and the sum of third order derivatives of $p_{1}$ (lower part).

As we mentioned above we were using FEMLAB package for our simulation. The boundaries of the computational domain $\Omega$ were constructed using standard tools 
of FEMLAB such like rectangles and arcs of circles. Hence, it is obvious that the curvature of the boundary $\partial \Omega$ is not continuous. Analyzing the results of the simulation we see that the third order derivatives of the function $p_{1}$ is not bounded near to the discontinuities of the curvature, cf. Fig. $3 b$ (lower part). This affects the behaviour of the functions $10 \log _{10}\left(\frac{\rho_{0}^{2} c_{0}^{4}}{2 p_{0}^{2}} \mu^{6}\left|p_{3}\right|^{2}\right)$ and $10 \log _{10}\left(\frac{\rho_{0}^{2} c_{0}^{4}}{2 p_{0}^{2}} \mu^{6}\left|q_{3}\right|^{2}\right)$. It might be, that in order to get smooth third order derivatives of $p_{1}$ we need continuous curvature of $\partial \Omega$.

As we promised in the previous section we show, that the wrong boundary conditions, namely homogeneous $\left.\frac{\partial p_{i}}{\partial \boldsymbol{n}}\right|_{\Gamma_{0}}=0, i=1,2,3$, on the rigid walls bring completely different results. The results of simulations are presented in Fig. 4. That means, that we always have to do proper analysis in order to determine the right boundary conditions. These conditions do not always vanish on $\Gamma_{0}$.

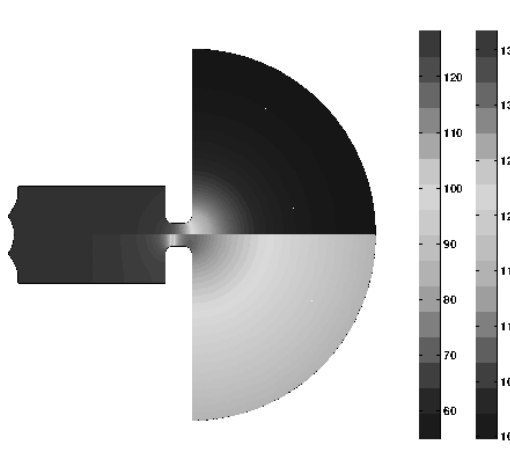

a)

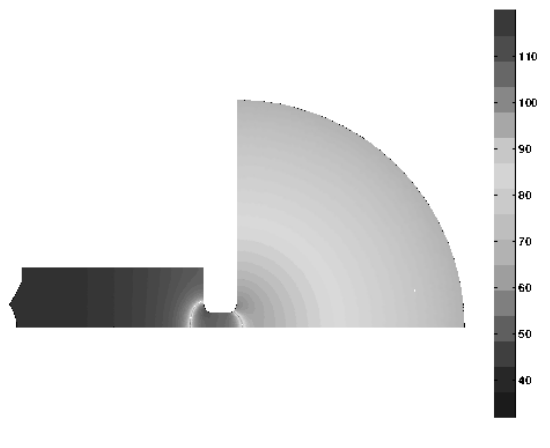

b)

Figure 4. a) Functions with wrong boundary conditions: $10 \log _{10}\left(\frac{\rho_{0}^{2} c_{0}^{4}}{2 p_{0}^{2}} \mu^{2}\left|p_{1}\right|^{2}\right)$ on the upper part (right color bar), $10 \log _{10}\left(\frac{\rho_{0}^{2} c_{0}^{4}}{2 p_{0}^{2}} \mu^{4}\left|p_{2}\right|^{2}\right)$ on the lower part (left color bar), b) the behaviour of $10 \log _{10}\left(\frac{\rho_{0}^{2} c_{0}^{4}}{2 p_{0}^{2}} \mu^{6}\left|p_{3}\right|^{2}\right)$ function with wrong boundary conditions.

\section{Conclusions}

In our work we started to consider classical Euler equations. In order to do linearization of non-linear Euler equations asymptotical analysis was applied. If a bass loudspeaker is run at high amplitudes we have to take into account not only linear part, but also second and even third order corrections. We derived, in addition to the results in [3], where the derivation of the second order correction to the classical Helmholtz equation was considered, the third order correction for the acoustical 
pressure function. This third order correction, as well as the second order correction, is of Helmholtz type with non-homogeneous free term. The technique, which is used in [3], was not applicable to get third order correction ((2.19) or (2.22)) because of the non-existence of the potential of $\boldsymbol{\eta}_{3}$ function. We applied another method to derive the appropriate equations, cf. subsection 2.3. At the same time we showed, that the assumption of an existence of the potentials of the functions $\boldsymbol{\eta}_{1}$ and $\boldsymbol{\eta}_{2}$ might be completely relaxed.

In order to complete the model rather simple concept of the boundary conditions was applied, cf. [2], [4]. Because the computational domain is axisymmetric we reduced our problem to the 2D case, cf. Fig. 1. The boundary in 2D consists of the straight lines and arcs of the circles. Because of this we paid our attention to the circle-shaped boundaries. However, similar concept of the boundary conditions could be applied for general boundaries, cf. [2]. These boundary conditions are of Neumann type and, in general, non-homogeneous on the rigid walls. The naive assumption of the homogeneous boundary conditions might lead completely to the different solution, cf. Fig. 3 - Fig. 4 and compare the results.

To get the numerical solution we used the FEMLAB package. We approximated the boundaries of our computational domain using straight lines and arcs. This leads to the discontinuous curvature what affect the results of the third order correction functions $p_{3}$ and $q_{3}$ because of the non-boundedness of third order derivatives of $p_{1}$ function. This is only a hypothesis. In our future work we will try to prove our apprehensions.

\section{References}

[1] F. Erwe. Differential- und Integralrechnung 2 Bde. B.I. Mannheim, 1988.

[2] J. Jegorovs. Optimal Shape of the Reflex Tube of a Bass Loudspeaker. Master Thesis, Fachbereich Mathematik, Universität Kaiserslautern, 2003.

[3] J. Mohring. Simulating Bass Loudspeakers Requires Nonlinear Acoustics - A Second Order Correction to the Helmholtz Equation. In: Progress in Industrial Mathematics at ECMI 2002, Springer-Verlag, Heidelberg, 333 - 339, 2002.

[4] J. Mohring and J. Jegorovs. Optimal Shape of the Reflex Tube of a Bass Loudspeaker. In: Proceedings of the International Conference on Noise and Vibration Engineering ISMA 2004, Leuven, Belgium, ID 111, 2004.

[5] M.L. Munjal. Acoustics of Ducts and Mufflers: with Application to exhaust and ventilation system design. John Wiley and Sons, Inc., 1987.

\section{Helmholco lygties trečiosios eilès patikslinimas}

J. Jegorovs, J. Mohring

Remiantis Oilerio lygtimis ir asimptotine analize gautas Helmholco lygties trečiosios eilès patikslinimas. Akustiniam slėgiui gauta Helmholco tipo lygtis bei jai išvestos sąlygos. Pateikti skaitinio modeliavimo rezultatai. 